\author{
UNIVERSIDADE DE SÃO PAULO \\ FACULDADE DE MEDICINA DE RIBEIRÃO PRETO
}

Renata da Silva Almeida Santos

\title{
Qualidade da Janela Transtemporal Definida pelo Ultrassom Transcraniano Colorido
}


Renata da Silva Almeida Santos

\section{Qualidade da Janela Transtemporal definida pelo Ultrassom Transcraniano Colorido}

Dissertação apresentada ao Departamento de Neurociências e Ciências do Comportamento da Faculdade de Medicina de Ribeirão Preto da Universidade de São Paulo para obtenção do título de Mestre em Ciências.

Área de concentração: Neurociências

Orientador: Prof. Dr. Octávio Marques Pontes Neto

Ribeirão Preto

2016 
AUTORIZO A REPRODUÇÃO E DIVULGAÇÃO TOTAL OU PARCIAL DESTE TRABALHO, POR QUALQUER MEIO CONVENCIONAL OU ELETRÔNICO, PARA FINS DE ESTUDO E PESQUISA, DESDE QUE CITADA A FONTE.

O exemplar original encontra-se no Departamento de Neurociências e Ciências do Comportamento da Faculdade de Medicina de Ribeirão Preto.

\section{FICHA CATALOGRÁFICA}

Santos, Renata da Silva Almeida

Qualidade da Janela Transtemporal Definida pelo Ultrassom Transcraniano Colorido, Ribeirão Preto, 2016.

47p. : il. ; $30 \mathrm{~cm}$

Dissertação de Mestrado apresentada à Faculdade de Medicina de Ribeirão Preto/USP. Área de concentração: Neurociências.

Orientador: Prof. Dr. Octávio Marques Pontes Neto

1. Acidente vascular cerebral. 2 Janela Transtemporal. 3. Ultrassonografia Transcraniana Colorida.4. Dupplex Transcraniano 


\section{FOLHA DE APROVAÇÃO}

Dissertação apresentada ao Departamento de Neurociências e Ciências do Comportamento da

Faculdade de Medicina de Ribeirão Preto da Universidade de São Paulo para obtenção do título de Mestre em Ciências.

Área de concentração: Neurociências

Aprovado em:

\section{BANCA EXAMINADORA}

Prof. Dr.

Instituição: Assinatura:

Prof. Dr.

Instituição: Assinatura:

Prof. Dr.

Instituição: Assinatura: 
DEDICATÓRIA

Dedico este trabalko cos meus pais Pegina o Silvio. 


\section{AGRADECIMENTOS}

Agradeço a todos que me acompanharam na realização deste sonho. Em especial, quero demonstrar meu profundo reconhecimento:

Agradeço ao Prof. Dr. Octavio Marques Pontes Neto, pelo voto de confiança, incentivo e competência demonstrados na orientação deste trabalho.

Aos neurologistas, Millene Rodrigues Camilo e Pedro Telles Cougo Pinto pela atenção, disponibilidade e orientações, fundamentais para este trabalho.

Aos colegas neurologistas Rui Kléber do Vale Martins Filho, Letícia Januzi Almeida Rocha, Milena Libardi, Clara Monteiro Barreira, Soraia Ramos Cabette Fábio, Francisco Antunes Dias e Frederico Fernandes Alessio Alves pela disponibilidade em contribuir pelos exames de Ultrassonografia Transcraniana Colorida e Doppler Transcraniano de todos os pacientes.

De forma muito especial, aos pacientes que participaram desta pesquisa que, mesmo diante de uma situação tão delicada, que é a internação hospitalar, se dispuseram a colaborar.

A todos, os meus sinceros agradecimentos! 


\title{
RESUMO
}

\author{
Santos, RSA. Qualidade da Janela Transtemporal Definida pelo Ultrassom \\ Transcraniano Colorido. 2016. 49f. Dissertação (Mestrado) - Faculdade de Medicina \\ de Ribeirão Preto, Universidade de São Paulo, Ribeirão Preto, 2016.
}

INTRODUÇÃO: O ultrassom transcraniano colorido (UTC) é um eficiente método para avaliação da circulação intracraniana e do fluxo sanguíneo cerebral em diversas condições clínicas incluindo o acidente vascular isquêmico agudo. Uma das principais limitação deste método reside na incapacidade de insonação intracraniana adequada através da janela transtemporal em até $20 \%$ dos pacientes. Neste contexto, surge a necessidade do desenvolvimento de uma metodologia estruturada que permitisse caracterizar a qualidade da janela transtemporal de forma mais detalhada e objetiva. No presente estudo, objetivamos: determinar a frequência de visualização dos principais marcos anatômicos pela insonação com UCT utilizando-se a janela transtemporal em pacientes com AVCI agudo; classificar o grau de dificuldade na visualização da primeira porção da artéria cerebral média (ACM) ipsilateral ao lado insonado; determinar a influência da idade, sexo, etnia no na qualidade da janela transtemporal pelo UTC. METODOLOGIA: Avaliamos prospectivamente todos os pacientes adultos, consecutivos, com o primeiro e único episódio de acidente vascular isquêmico no período de julho de 2014 a janeiro de 2015 com um exame de UTC (modo B e Doppler colorido). Dois examinadores classificaram a qualidade da janela transtemporal pelo modo B utilizando uma escore baseado na qualidade da visualização de referencias anatômicas (osso temporal contralateral, asa menor do esfenoide ipsilateral e mesencéfalo) variando de 0 (janela ausente) a 9 (excelente janela). Os preditores independentes de uma boa visualização do sinal da ACM ao UTC foram identificados através de um modelo de regressão logística multivariada selecionado pelo método backward. A acurácia do escore do modo B foi avaliada através dos parâmetro de sensibilidade, especificidade e estatística C (curva ROC). RESULTADOS: entre os 200 paciente incluídos no estudo (55\% do sexo masculino e com idade média de $61,17 \pm 15,22$ anos) o osso temporal contralateral não foi visualizado em $48,5 \%$ dos casos, o mesencéfalo foi visualizado em $65 \%$ e a asa menor do esfenoide foi visualizada em $66 \%$. A porção proximal da artéria cerebral media (M1) foi visualizada em 62\% dos casos. Na análise multivariada, a idade (OR: 0,95; IC95\%:0,92 $0,99 ; \mathrm{p}=0,011)$ e a pontuação no escore do modo B (OR: 2,97; IC95\%:1,93- 4,55; p<0,001) foram preditores independentes de visualização do sinal da ACM ao UTC. A pontuação no escore do modo B mostrou um preditor acudado para subsequente visualização da artéria cerebral médica pelo Doppler colorido com uma área sob a curva ROC de 0,896 ( $<<0,001)$. $\mathrm{O}$ escore de 2 apresentou uma sensibilidade e especificidade de 80 e $87 \%$ para este fim, respectivamente. CONCLUSÃO: $O$ escore do modo B baseado na visualização de referência anatômicas intracranianas pelo modo B do UTC é uma ferramenta útil com boa acurácia para capacidade de visualização do fluxo da artéria cerebral media ipsilateral ao UTC. Este escore permite descrever de forma mais detalhada a qualidade de janela transtemporal ao UTC, em suas diferentes aplicações.

Palavras-chave: Acidente vascular cerebral; Ultrassonografia Transcraniana Colorida; Doppler Transcraniano. 


\begin{abstract}
Santos, RSA. Quality Assessment for the Transtemporal Window by Transcranial Color-Coded Sonography. 2016. 49f. Dissertação (Mestrado) - Faculdade de Medicina de Ribeirão Preto, Universidade de São Paulo, Ribeirão Preto, 2016.
\end{abstract}

INTRODUCTION: Transcranial Color-Coded Sonography (TCCS) is an widely-used method to assess the intracranial circulation and cerebral blood flow in several clinical conditions including acute ischemic stroke. One of the main limitations of this technic is related to the quality of the transtemporal window, which is poor in about $20 \%$ of cases. In this context, it is important to develop an structured approach to better define the quality of the transtemporal window. In this study we aimed to evaluate how frequently the main anatomic landmarks can be adequately visualized by TCCS in acute stroke patients using the transtemporal window; to assess the proportion of patients in which the ipsilateral middle cerebral artery of visualized by TCCS; and to identify the main predictors of a poor transtemporal window on TCCS. METHODS: We examined 200 consecutive acute ischemic stroke (AIS) patients, from July 2014 to January 2015. All patients underwent prospective TCCS evaluation. Visualization of the contralateral temporal bone (CTB), midbrain (MB) and lesser sphenoid wing (LSW) was scored on B-mode images. The resulting B-Mode Score varied from 0 (poor visualization) to 9 (perfect window). A multivariate logistic regression model (backward selection) was used to identify the independent predictors of visualization of the MCA signal by TCCS. The relationship between these B-Mode Score and visualization of the proximal portion of the ipsilateral MCA was assessed in terms of sensitivity, specificity, ROC curve and C statistics. RESULTS: Among 200 AIS patients (male 55\%; mean age, $61.17 \pm 15,22$ years), CTB was invisible' in $48,5 \%$, the MB was visualized in $65 \%$; and the LSW was seen in $66 \%$. The M1 segment of the MCA was detected in $62 \%$ of cases. After multivariate analysis, age (OR: 0.95; 95CI\%:0.92-0.99; $\mathrm{p}=0.011$ ) and B-Mode score (OR: 2.97;95CI\%:1.93-4.55; $\mathrm{p}<0.001)$ were independente predictors of visualization of MCA signal by TCCS. The BMode Score show good accuracy for the prediction of MCA visualization with an AUC of 0,896. $(p<0,001)$ on the respective ROC curve. A cut-off of 2 on the B-mode score showed a sensitivity of $80 \%$ and a specificity of $87 \%$ for adequate MCA visualization by TCCS. CONCLUSION: The B-mode Score, which is based on the visualization of intracranial anatomical landmarks on B-Mode, appears to be a reliable way to characterize the quality of the transtemporal window, with a good accuracy as predictor for visualization of the ipsilateral MCA on TCCS. This score may allow more detailed characterization of the transtemporal window for different clinical applications of TCCS.

Keywords: Stroke, temporal window, Transcranial Color Coded Sonography, Transcranial Doppler. 


\section{LISTA DE TABELAS}

Tabela 1: Características basais dos pacientes estudados

Tabela 2: Análise univariada dos preditores de visualização do fluxo de artéria cerebral média pelo Doppler colorido.

Tabela 3: Frequência de visualização das referências anatômicas no modo B de acordo com o gênero.

Tabela4: Frequência da categorização das variáveis ao modo B, osso temporal, mesencéfalo, asa do esfenóide, na população estudada.

Tabela 5: Análise univariada para preditores de ACM visualizável.

Tabela 6: Análise multivariada para preditores independentes de ACM visualizável no duplex transcraniano.

Tabela 7: Análise multivariada para preditores independentes de ACM visualizável no duplex transcraniano incluindo o Escore do Modo B.

Tabela 8: Área sob a curva relacionada a curva ROC de preditores da visualização do fluxo na artéria cerebral média. 


\section{LISTA DE FIGURAS}

Figura 1: Apresenta sinal ótimo do osso temporal contralateral (3), sinal insatisfatório da asa do esfenóide (0) e sinal insatisfatório do mesencéfalo (0).

Figura 2: Apresenta sinal ótimo do osso temporal contralateral (3), sinal ótimo da asa menor do esfenóide (3), sinal ótimo do mesencéfalo (3).

Figura 3: Apresenta sinal ótimo do osso temporal contralateral (3), sinal pobre da asa menor do esfenóide (1) e sinal insatisfatório do mesencéfalo (0).

Figura 4: Apresenta sinal pobre do osso temporal contralateral (1), sinal pobre da asa menor do esfenóide (1), sinal insatisfatório do mesencéfalo (0).

Figura 5: Apresenta sinal satisfatório do osso temporal contralateral (2), sinal satisfatório da asa menor do esfenóide (2), sinal insatisfatório do mesencéfalo (0).

Figura 6: Sinal de fluxo da artéria cerebral média ipsilateral ao Doppler colorido. Figura 7: Análise de sensibilidade para determinação do ponto operador ótimo para o escore do modo B como preditor da visualização do sinal do fluxo da artéria cerebral média no Doppler colorido.

Figura 8: Análise de sensibilidade para determinação do ponto operador ótimo para o escore do modo B como preditor da visualização do sinal do fluxo da artéria cerebral média no Doppler colorido. 


\section{ABREVIATURAS}

ACM: Artéria cerebral média

ACA: artéria cerebral anterior

ACP: artéria cerebral posterior

AIT: Ataque Isquêmico Transitório

AVCi: Acidente vascular cerebral isquêmico

DCV: doenças cerebrovasculares

DM: Diabetes Mellitus

DTC: Doppler Transcraniano

HAS: hipertensão arterial sistêmica

HCFMRP-USP: Hospital das Clínicas da Faculdade de Medicina de Ribeirão Preto da Universidade de SãoPaulo

HITS: do Inglês, high intensity transitory signs

IC: intervalo de confiança

IQ: intervalo interquartil

JTT: janela transtemporal

MHz: megahertz

NIHSS: National Institutes of Health Stroke Scale

OR: odds ratio

PA: pressão arterial

RM: Ressonância Magnética

SUS: Sistema Único de Saúde

TC: Tomografia Computadorizada

TCCS: do Inglês, Transcranial Color Coded Ultrasound

UTC: UltrassomTranscraniano Colorido

VS: velocidade sistólica

VD: velocidade diastólica

VM: velocidade média 


\section{SUMÁRIO}

1. INTRODUÇÃ

1.1 Doppler Transcraniano.................................................................................. 13

1.2 Ultrassonografia Transcraniana Colorida ............................................................... 15

1.3 Justificativa do estudo....................................................................................... 16

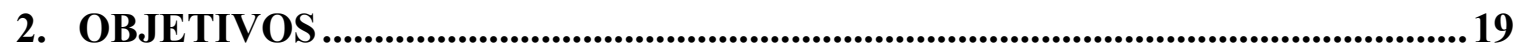

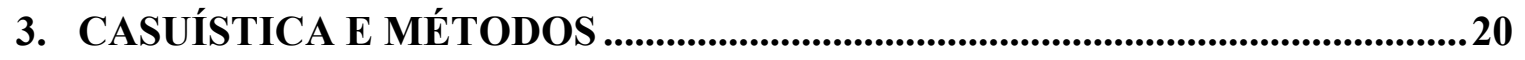

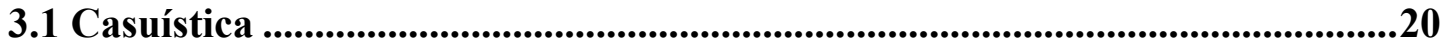

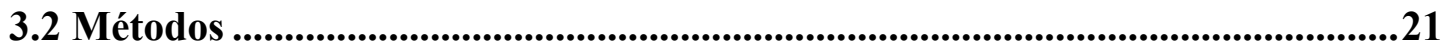

3.2.1 - Exame de Ultrassom Transcraniano Colorido.................................. 22

3.2.2 - Descrição da técnica do exame.............................................................. 22

3.2.3 - Análise Estatística .........................................................................26

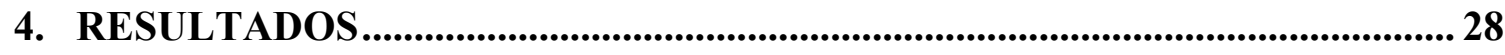

4.1 - Características dos pacientes com AVC incluídos no estudo........................ 28

4.2 - Achados ao modo B Escore do modo B.............................................................. 31

4.3 - Avaliação da acurácia do modo B. ..........................................................................34

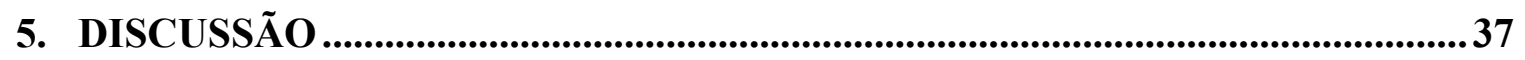

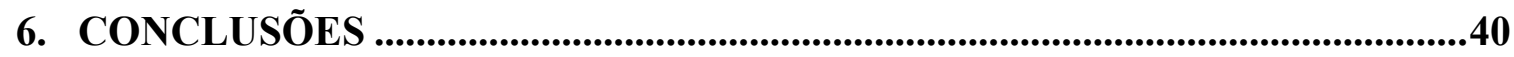

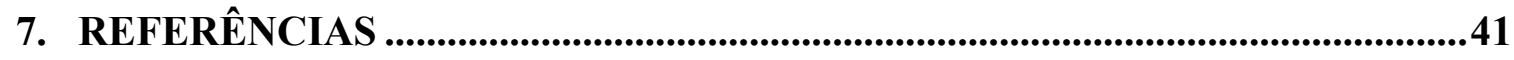

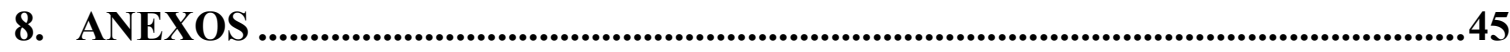




\section{Introdução}

\section{$1.1 \quad$ Doppler Transcraniano}

O Doppler transcraniano (DTC) foi introduzido na prática clínica em 1982 por Rune Aaslid. É um método não-invasivo baseado na energia do ultrassom, em que a informação obtida pela emissão pulsada de ondas sonoras de baixa frequência através do crânio e processada por algoritmos matemáticos e transformada em mapas dinâmicos de frequência que nos permitem avaliar de forma indireta a velocidade de fluxo da circulação intracraniana em diversas condições fisiológicas e patológicas. ${ }^{1-3}$

O DTC é um exame de baixo custo que pode ser usado à beira-do-leito, na fase aguda do acidente vascular cerebral para diagnosticar oclusão arterial, estenose intracraniana, avaliar a repercussão hemodinâmica de estenoses extracranianas e monitorizar recanalização arterial após terapia trombolítica. Também auxilia na monitorização de vasoespasmo cerebral, hipertensão intracraniana, vasorreatividade cerebral, microembolismo cerebral e no diagnóstico de parada circulatória cerebral. Em nível ambulatorial, o DTC contribui para a orientação terapêutica na indicação de transfusões sanguíneas de repetição em crianças com anemia falciforme. ${ }^{4}$

Durante o exame de DTC, uma sonda aplicada ao crânio emite ondas ultrassônicas (geralmente com freqüência de $2 \mathrm{Mhz}$ ) que atravessam o crânio e são refletidas pelas diferentes estruturas intracranianas, incluindo as células sanguíneas em movimento dentro dos vasos cerebrais. Estas ondas refletidas retornam ao transdutor do aparelho de DCT e a partir daí, um algoritmo matemático é aplicado a informação captada pela sonda, gerando mapas de frequências que permitem estimar parâmetros hemodinâmicos cerebrais tais como: velocidade sistólica (VS), velocidade diastólica (VD), velocidade média (VM), 
índice de pulsatilidade (VS-VD/ VM) e índice de resistência (VS-VD/VS) do fluxo sanguíneo nos casos cerebrais. Estes dados são geralmente apresentados na tela do computador acompanhados de uma análise espectral do fluxo no vaso insonado. ${ }^{5}$

A insonação dos vasos intracranianos acontece através de janelas ósseas ou janelas acústicas do crânio. Estas janelas são forames naturais do crânio em que há mais facilidade de penetração da onda sônica para acesso à vasculatura intracraniana.

São três as janelas acústicas clássicas para o DTC:

- janela transtemporal, descrita por Aaslid em $1982^{3}$

- janela transorbitária, descrita por Spencer e Whisler em $1983^{7}$

- janela transforaminal, descrita por Arnolds e von Reutern em $1986^{7}$

A janela transtemporal está localizada no osso temporal, logo acima do arco zigomático. Corresponde à camada escamosa, mais fina do osso temporal. Pode apresentar atenuação variável dependendo da espessura óssea e de sua composição. ${ }^{3,7}$ De forma que, utilizando-se a insonação pela janela transtemporal, a intensidade acústica do ultra-som conduzido no tecido cerebral é $35 \%$ menor do que a intensidade acústica original. Classicamente, a janela transtemporal pode ser dividida em quatro áreas distintas: posterior, média, anterior e frontal. ${ }^{3}$

Os vasos estudados através da janela transtemporal de cada lado insonado são essencialmente: a parte distal da artéria carótida interna, a artéria cerebral anterior (ACA), cerebral média $(\mathrm{ACM})$, a artéria comunicante anterior, cerebral posterior $(\mathrm{ACP})$ e o topo da artéria basilar. ${ }^{3,5,7}$

A janela transorbital, por sua vez, permite a transmissão da onda de ultra-som através da fina superfície orbital do osso frontal, canal ótico e/ou fissura orbital superior e permite acessar o fluxo sanguíneo na artéria carótida interna e na artéria oftálmica. ${ }^{4-7}$ 
Ja a janela transforaminal permite o acesso às porções intracranianas das artérias vertebrais e artérias basilares através do forame natural, aberto entre o crânio e o atlas. ${ }^{4-7}$

\subsection{Ultrassonografia Transcraniana Colorida}

O ultrassom transcraniano colorido (UTC) é um método mais recente de ultrassonografia transcraniana que associa a imagem bidimensional (Modo B) ao sinal do Doppler, o que implica em algumas vantagens em relação ao DTC convencional. ${ }^{8}$ Essencialmente, o UTC é um método mais específico para identificar referências anatômicas intracranianas, e assim identificar o segmento vascular intracraniano insonado, variações anatômicas na vasculatura intracraniana, assim como identificar mais prontamente segmentos com alteração nas velocidades de fluxo, além de oferecer a possibilidade de correção do ângulo insonação. ${ }^{9}$ Outra vantagem potencial deste método, quando comparado ao DTC, é a possibilidade de realização de exames utilizando-se aparelhos convencionais de ultrassonografia comercialmente disponíveis, através de um transdutor de $2 \mathrm{Mhz}$ acoplado, utilizando-se um software com aplicação para circulação intracraniana, eliminando assim a necessidade de aquisição de uma aparelho exclusivo para DTC. ${ }^{10}$ Desta forma, a disponilidade do UTC é maior e o custo de aquisição acaba sendo menor do que o DTC, que necessita transdutores e aparelho dedicados. Por outro lado as janelas utilizadas para o exame de UTC são essencialmente as mesmas usadas com o DTC.

Sabe-se, entretanto, que entre 5 a $37 \%$ da população não se consegue sucesso na insonação da janela transtemporal pelo DTC devido a absorção excessiva e dispersão da intensidade do sinal sonográfico através do osso temporal. Diversos estudos reportam uma dificuldade maior em insonar a janela transtemporal pelo DTC em mulheres, não caucasianos e idosos. $^{11}$ 
Existem poucos estudos entretanto sobre a eficiência do UTC para visualização de estruturas e avaliação do fluxo sanguíneo na vasculatura intracraniana nas diferentes etnias. $^{5-7}$ Até o momento estudos sugerem uma maior taxa de sucesso na população do norte europeu, e menor taxa de sucesso em asiáticos, afro-americanos e hispânicos. ${ }^{2,12,13}$

Neste contexto, surge a necessidade de conhecer melhor as limitações do exame de UTC para visualização de estruturas intracranianas em nosso meio, e sistematizar de forma mais detalhada e quantitativa a qualidade da janela transtemporal em nosso meio. Este tipo de quantificação teria impacto na prática clínica, facilitando utilização adequada do método em situações de emergência, como durante o manejo do AVC hiperagudo, mas também fornecendo mais confiabilidade ao método em situações onde a sua especificidade é indispensável, como no diagnóstico de parada circulatória cerebral ${ }^{14}$.

\subsection{Justificativa para o presente estudo}

Conforme elencado acima, a qualidade da janela transtemporal ainda é a principal limitação para aplicação ampla do UTC, como método portátil de baixo custo e de baixíssimo risco para avaliação do fluxo sanguíneo cerebral em suas diversas aplicações clínicas. Na grande maioria dos estudos científicos e na prática clinica, a qualidade da janela temporal tem sido classificada de forma muito subjetiva e binária (boa ou ruim; presente ou ausente). Por tratar-se de um método examinador dependente, esta subjetividade pode trazer imprecisão à aplicação do método e tem impacto negativo importante sua acurácia, sobre tudo em situações onde a sensibilidade e especificidade do 
método são críticas para a tomada de decisão como no manejo do paciente com AVC agudo ou no diagnóstico de parada circulatória cerebral.

No presente estudo, objetivamos avaliar a eficiência do UTC na identificação de marcos anatômicos intracranianos pelo modo B, como forma de classificar e definir de forma semi-quantitativa a qualidade da janela transtemporal para o estudo do fluxo na ACM ipsilateral em pacientes com acidente vascular cerebral. Além disso, pretendemos desenvolver e testar a acurácia de um score prático de qualidade da janela transtemporal no modo B como forma de classificar de forma semi-quantitativa a qualidade da janela transtemporal para avaliação do fluxo sanguíneo da ACM ipsilateral nestes pacientes. 


\section{Objetivos}

1 - Determinar a frequência da visualização através do UTC de marcos anatômicos ultrassonográficos intracranianos, quais sejam, osso temporal contralateral, mesencéfalo, asa menor do osso esfenóide, em pacientes admitidos por suspeita de acidente vascular cerebral.

2 - Avaliar a distribuição e acurácia de um escore (escore do modo B) que relacione a qualidade da visualização de marcos anatômicos pelo modo B com a capacidade de insonação adequada da ACM ipsilateral.

3 - Identificar os fatores preditores independentes de boa janela para visualização do fluxo sanguíneo na ACM ipsilateral. 


\section{Casuística e Métodos}

\section{1 - CASUÍSTICA}

Este estudo foi realizado de forma prospectiva com uma amostra populacional de pacientes registrados e admitidos na Unidade de Emergência do HCFMRP-USP. Todos os pacientes, no momento da inclusão no estudo, tinham a suspeita de Doença Cerebrovascular (DCV) isquêmica aguda, quais sejam, Acidente Vascular Cerebral isquêmico (AVCi ) ou Ataque Isquêmico transitório (AIT).

O estudo foi conduzido de acordo com a Declaração de Helsinki (1975) e a Resolução $n^{0}$ 466, do Conselho Nacional de Saúde, do Ministério da Saúde do Brasil (2012). O protocolo de estudo foi aprovado pelo Comitê de Ética em Pesquisa (CEP) do Hospital das Clínicas da Faculdade de Medicina de Ribeirão Preto (HCFMRP - USP): número CAAE: 32115414.0.0000.5440 (Anexo A). O Termo de Consentimento Livre e Esclarecido foi obtido de cada paciente ou seu representante legal antes da inclusão no estudo.

Foram avaliados, prospectivamente, os pacientes admitidos na Unidade de Emergência do HCFMRP-USP, de acordo com os critérios de inclusão e exclusão abaixo listados no período de Julho de 2014 a Janeiro 2015.

Estabeleceu-se como critério de inclusão: paciente admitido com suspeita de acidente vascular cerebral agudo ou ataque isquêmico transitório, assinatura do termo de consentimento pelo paciente ou responsável legal.

Os critérios de exclusão foram:

- Pacientes submetidos à craniectomia descompressiva ou qualquer procedimento 
neurocirúrgico prévio;

- Instabilidade hemodinâmica no momento da realização do exame ultrassonográfico;

- Pacientes com oclusão bilateral da ártéria cerebral média detectada por qualquer método de neuroimagem.

- Não assinatura do termo de consentimento informado de acordo com comitê de ética do HCFMRP-USP.

\section{2 - MÉTODOS}

Trata-se de um estudo observacional, analítico, de corte transversal. No período da pesquisa, foram incluídos 200 pacientes com suspeita de AVC de julho de 2014 a janeiro de 2015, na Unidade de Emergência do HCFMRP-USP.

No período da internação hospitalar, esses pacientes foram submetidos à investigação da etiologia do AVCi ou AIT conforme protocolo institucional que inclui: exame de imagem de admissão e controle com definição de território isquêmico (Tomografia Computadorizada de Crânio e/ou Ressonância Magnética Cerebral), estudo de vasos intracranianos e cervicais ( Angiotomografia e/ou Angiorresonância e/ou Doppler Cervical, DTC e/ou UTC), exames laboratoriais ( lipidograma, função renal, glicemia, hemoglobina glicosilada, enzimas hepáticas, enzimas cardíacas, eletrólitos, sorologias para doença de Chagas e Sifílis).

Os exames de UTC foram realizados em todos os pacientes por examinadores treinados em UTC na Unidade de Emergência da instituição. Um subgrupo de 20 pacientes 
foi avaliado por dois examinadores para a determinação da concordância entre examinadores para os achados relacionados ao escore do Modo B (kappa de Cohen).

\subsubsection{Descrição do modelo de equipamento utilizado: UTC}

Para a definição da qualidade da janela transtemporal foi utilizado um equipamento de ultrassonografia da marca Toshiba Xario modelo SSA- 660A, equipado com transdutor de $2 \mathrm{MHz}$ (tipo phase-array).

\subsection{2 - Descrição das etapas do estudo}

1. Admissão de paciente com suspeita de doença cerebrovascular isquêmica aguda.

2. Realização de exame neurológico, Tomografia Computadorizada de crânio, eletrocardiograma e exames laboratoriais.

3. Instituição de condutas clínicas para manejo do evento isquêmico agudo.

4. Leitura, concordância e assinatura do "Termo de Consentimento Livre e Esclarecido”, aprovado pelo Comitê de Ética do HCFMRP (Anexo E)

5. Preenchimento de uma ficha padronizada contendo os dados demográficos dos pacientes (idade, sexo, etnia/cor da pele) e dados referentes aos fatores de risco para doença vascular: hipertensão arterial sistêmica (HAS), diabetes mellitus (DM), tabagismo, etilismo, dislipidemia, histórico pessoal de neurocirurgia com craniectomia.

6. Realização do UTC.

\subsection{3 - Técnica de realização do exame: UTC}

Os exames de UTC foram realizados de acordo com protocolo institucional que inclui estudo dos vasos cervicais e intracranianos para investigação etiológica do evento 
cerebrovascular. Na primeira parte do exame de UTC, procedeu-se a avaliação da qualidade da janela transtemporal realizada por um protocolo estruturado.

Os exames foram armazenados em formato DICOM e JPEG para posterior avaliação e classificação cega da janela pelos pesquisadores. O protocolo de avaliação da janela transtemporal incluía a seguintes fases: insonação através da janela transtemporal de um lado de cada vez em plano axial, registro em JPEG e DICOM estruturas anatômicas visíveis ao modo B: asa do esfenóide, mesencéfalo, osso temporal contralateral em plano axial.

A artéria cerebral média foi identificada ao UTC no ponto em que ela é geralmente visualizada no monitor após o sifão carotídeo e a porção distal da artéria carótida interna, ao longo da borda lateral da asa menor do esfenoide. Os exames foram realizados bilateralmente em cada pacientes, sendo que o lado com melhor visibilidade foi utilizado para aplicação do escore. Em casos de oclusão conhecida ou posteriormente detectada unilateral da artéria cerebral média, o lado não ocluído foi utilizado para aplicação do escore.

\subsubsection{Descrição das etapas de realização do UTC}

1. Paciente em decúbito dorsal, hemodinamicamente estável com ou sem medicação vasoativa.

2. Posicionamento do trasndutor acima do arco zigomático.

3. Registro das estruturas insonadas ao modo B, quais sejam, osso temporal contralateral, asa do esfenóide e mesencéfalo.

4. Registro do sinal da artéria cerebral média ao modo colorido.

A gradação da visualização das três referências anatômicas (asa menor do esfenoide ipsilateral, osso temporal contralateral e mesencéfalo) - ao modo B foi realizada em um segundo momento por um único examinador após a realização do exame de UTC e de 
forma cega para a presença ou ausência de visualização da artéria cerebral média ipsilateral. Para esta gradação da visualização da asa menor do esfenoide ipsilateral e do osso temporal contralateral foi utilizada a seguinte categorização:

0 - Sinal insatisfatório: nenhum sinal sonográfico detectável

1 - Sinal pobre: sinal sonográfico menor que $50 \%$ da estrutura

2 - Sinal satisfatório: sinal sonográfico maior que $50 \%$ da estrutura

3 - Sinal ótimo: sinal sonográfico em $100 \%$ do contorno da asa estrutura

A gradação da interpretação do sinal do mesencéfalo ao modo B foi realizada com a seguinte categorização:

0 - Sinal insatisfatório: nenhum sinal sonográfico detectável

1 - Sinal pobre: sinal sonográfico menor que $50 \%$ do mesencéfalo

2 - Sinal satisfatório: sinal sonográfico maior que 50\% do mesencéfalo

3 - Sinal ótimo: sinal sonográfico em $100 \%$ do contorno do mesencéfalo

Após a gradação da interpretação do sinal da asa do esfenóide, do mesencéfalo e do osso temporal contralateral foi criado um escore, denominado escore modo B, cuja pontuação varia de zero a nove. Ao escore modo $\mathrm{B}$, foi então relacionado a presença ou não do sinal da artéria cerebral média no exame de Doppler Colorido.

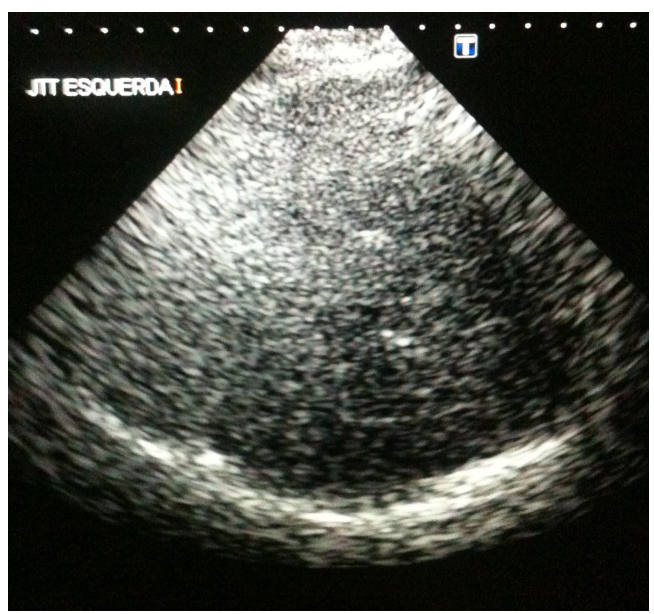

Figura 1: Apresenta sinal ótimo do osso temporal contralateral (3), sinal insatisfatório da asa do esfenóide (0) e sinal insatisfatório do mesencéfalo (0). 


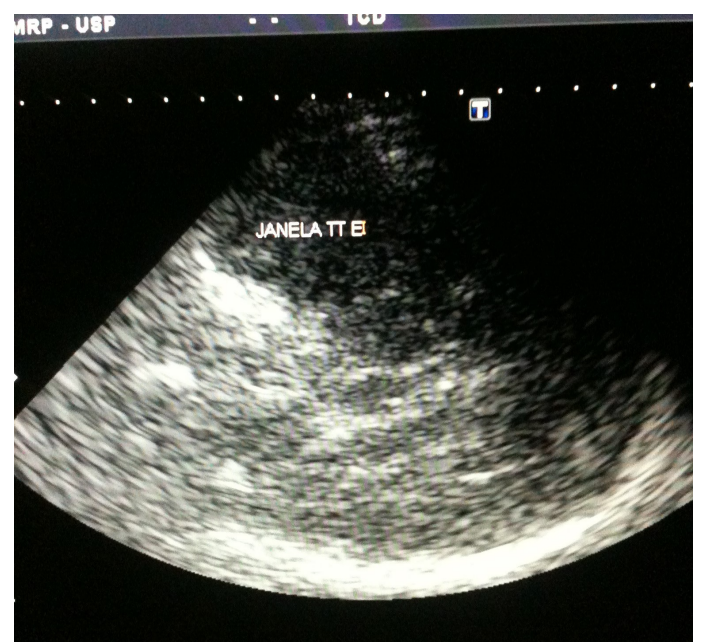

Figura 2: Apresenta sinal ótimo do osso temporal contralateral (3), sinal ótimo da asa menor do esfenóide (3), sinal ótimo do mesencéfalo (3). 


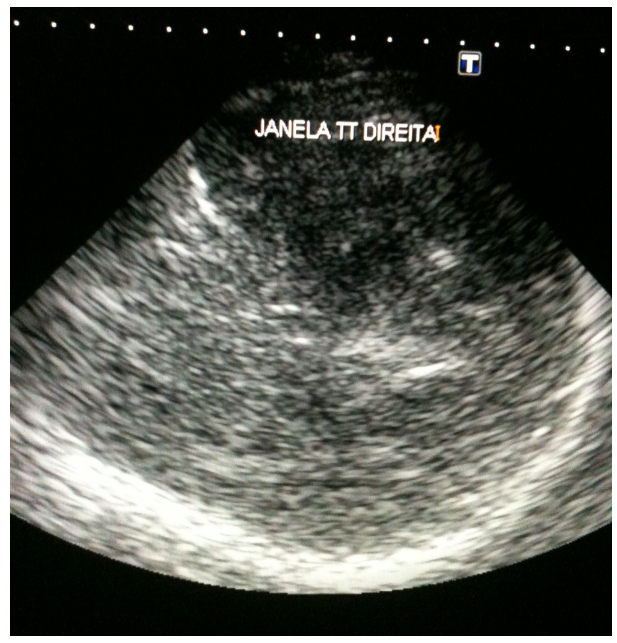

Figura 3: Apresenta sinal ótimo do osso temporal contralateral (3), sinal pobre da asa menor do esfenóide

(1) e sinal insatisfatório do mesencéfalo (0).

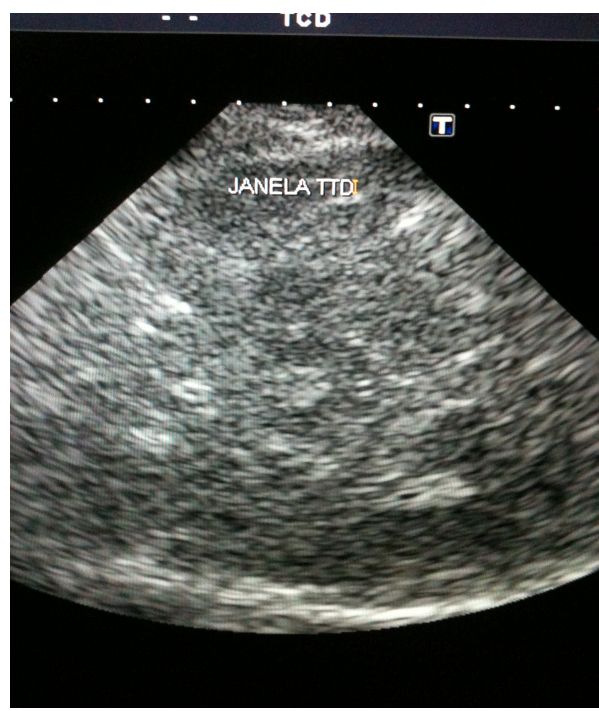

Figura 4: Apresenta sinal pobre do osso temporal contralateral (1), sinal pobre da asa menor do esfenóide (1), sinal insatisfatório do mesencéfalo (0). 


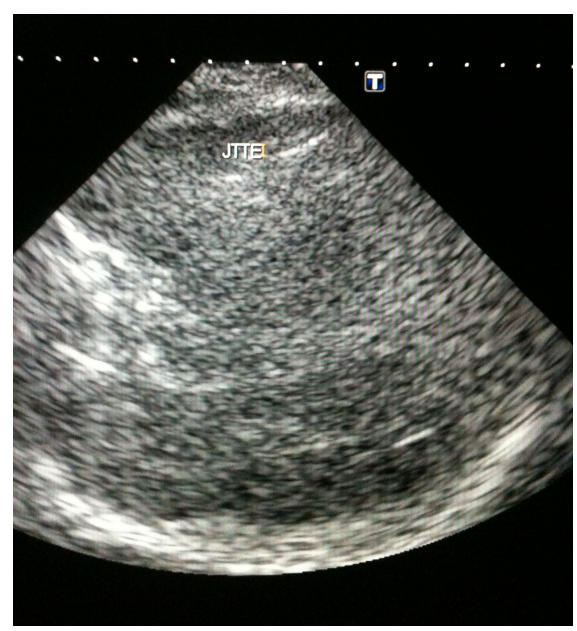

Figura 5: Apresenta sinal satisfatório do osso temporal contralateral (2), sinal satisfatório da asa menor do esfenóide (2), sinal insatisfatório do mesencéfalo (0).

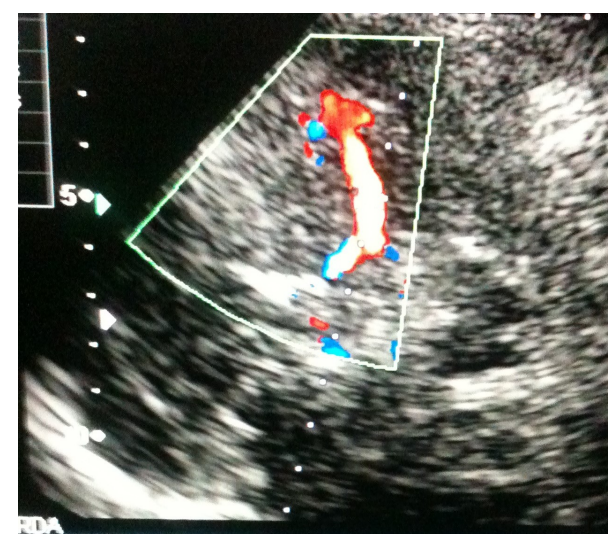

Figura 6: Presença de sinal da artéria cerebral média

\subsection{5 - Análise estatística}

Para a análise proposta neste estudo foram coletadas as seguintes variáveis: idade (anos), sexo, cor da pele, principais fatores de risco cerebrovasculares (entre eles: hipertensão arterial, diabetes, dislipidemia), qualidade da visualização de referências anatômicas intracranianas pelo modo B do UTC (osso temporal contralateral, asa menor 
do esfenóide ipsilateral, mesencéfalo), sinal de fluxo sanguíneo na topografia da ACM ipsilateral pelo Doppler colorido e o Escore do Modo B.

Para a análise estatística descritiva foram utilizadas medidas de tendência central (média e mediana) e dispersão (desvio padrão e intervalo interquartil) para variáveis numéricas. Variáveis categóricas foram descritas pela moda e frequências em números absolutos e em porcentagem. A estatística inferencial foi realizada através do Teste Exato de Fisher, teste Qui-Quadrado para variáveis categóricas e teste t-student para variáveis contínuas e comparativa das proporções entre as variáveis qualitativas. Regressão logística foi utilizada na análise multivariada para identificação dos preditores independentes de visualização do sinal da ACM ao UTC. O modelo final de regressão logística foi selecionado pelo método backward. O nível de significância estatística para todas as análises foi de $\mathrm{p}<0,05$ (bicaudal).

A acurácia do escore do modo $\mathrm{B}$ como preditor da visualização do fluxo na ACM ipsilateral foi avaliada por uma curva ROC e estatística C. A área sobre a curva foi avaliada assim como sua significância estatística. Diferentes valores de ponto de corte foram testados como preditores considerando a sensibilidade, especificidade, valor preditivo positivo e valor preditivo negativo, razão de verossimilhança (positiva, negativa) e cálculos das probabilidades pré e pós teste. O Programa estatístico utilizado para esse estudo foi o SPSS versão 20 (IBM INC., ARMONK, NY,EUA). 


\section{Resultados}

\section{1 - Características dos pacientes com AVC incluídos no estudo}

Durante o período de realização do estudo (julho de 2014 a janeiro de 2015), foram incluídos 200 pacientes.

As principais características basais dos pacientes admitidos, segundo critérios de inclusão e exclusão, estão descritas na tabela 1. Esta população foi constituída, na sua maioria, por homens 110 (55\%). A idade média foi de 61,17 $\pm 15,22$ anos, com predomínio de indivíduos de pele branca (159 pacientes - 79,5\%).

Com relação aos fatores de risco cardiovasculares, o mais frequentemente observado foi a hipertensão arterial sistêmica, identificada em 162 pacientes (81\%). A dislipidemia esteve presente em $117(58,5 \%)$ dos pacientes e diabetes melito em 57 pacientes $(28,5 \%)$. 
Tabela 1: Características basais dos pacientes estudados, $\mathrm{N}=200$.

\begin{tabular}{|lc|}
\multicolumn{1}{|c}{ Características } & $\mathrm{N}=200$ \\
\hline Idade, anos ${ }^{*}$ & $61,17 \pm 15,22$ \\
\hline Cor da pele & $159(79,5 \%)$ \\
Brancos & $41(21,5 \%)$ \\
\hline Sexo Masculino e pardos & $110(55 \%)$ \\
\hline Hipertensão arterial & $162(81 \%)$ \\
\hline Diabetes melito & $57(28,5 \%)$ \\
\hline Dislipidemia & $117(58,5 \%)$ \\
\hline
\end{tabular}

${ }^{*}$ Dados expressos em média \pm desvio padrão.

A análise univariada para identificar as características demográficas, clinicas e do Modo B relacionadas a visualização adequada do fluxo na artéria cerebral médica revelou que o sexo feminino, idade elevada, a visualização de qualquer uma das estruturas anatômicas de referencia (osso temporal contralateral, asa menor do esfenoide ou mesencéfalo) pelo modo B ou o escore do Modo B foram associados a janela transtemporal adequada para insonação vascular (tabela 2). 
Tabela 2. Análise univariada dos preditores de visualização do fluxo de artéria cerebral média pelo Doppler colorido.

\begin{tabular}{|c|c|c|c|}
\hline \multirow[t]{2}{*}{ Características } & \multicolumn{2}{|c|}{$\begin{array}{l}\text { Visualização da Artéria } \\
\text { Cerebral Média }\end{array}$} & \multirow[t]{2}{*}{ P-valor } \\
\hline & Ausente & Presente & \\
\hline \multicolumn{4}{|l|}{ Genero } \\
\hline Feminino & $32(78,05)$ & $58(36,48)$ & $<0,0001$ \\
\hline Masculin & $9(21,95)$ & $101(63,52)$ & \\
\hline \multicolumn{4}{|l|}{ Hipertensão } \\
\hline Não & $5(12,20)$ & $33(20,75)$ & 0.2129 \\
\hline Sim & $36(87,80)$ & $126(79,25)$ & \\
\hline \multicolumn{4}{|l|}{ Diabetes } \\
\hline Não & $29(70,73)$ & $114(71,70)$ & 0.9027 \\
\hline Sim & $12(29,27)$ & $45(28,30)$ & \\
\hline \multicolumn{4}{|c|}{ Asa menor do Esfenoide } \\
\hline Não & $38(92,68)$ & $28(17,61)$ & $<0,0001$ \\
\hline Sim & $3(7,32)$ & $131(82,39)$ & \\
\hline \multicolumn{4}{|l|}{ Mesencefalo } \\
\hline Não & $37(90,24)$ & $33(20,75)$ & $<0,0001$ \\
\hline Sim & $4(9,76)$ & $126(79,25)$ & \\
\hline \multicolumn{4}{|l|}{ Osso temporal } \\
\hline Não & $34(82,93)$ & $63(39,62)$ & $<0,0001$ \\
\hline Sim & $7(17,07)$ & $96(60,38)$ & \\
\hline Escore Modo B & $0(0-0)$ & $3(2,0-7,0)$ & $<0,0001$ \\
\hline Idade & $0,54(12,71)$ & $58,75(14,91)$ & $<0,0001$ \\
\hline
\end{tabular}




\section{2 - Achados ao modo B e Escore do modo B}

Na tabela abaixo (tabela 3), encontra-se a frequência de visualização das estruturas intracranianas, quais sejam, osso temporal, asa do esfenóide, mesencéfalo, na população estudada, de acordo com o gênero. Nela se observa que as mulheres apresentaram menor média de idade, no entanto, maior frequência de categorização de sinal insatisfatório dos referenciais anatômicos.

Tabela 3. Frequência de visualização das referências anatômicas no modo B de acordo com o gênero.

\begin{tabular}{|llllll|}
\hline & Idade & Temporal & Esfenóide & Mesencéfo ACM \\
\hline Feminino (n=90) & 60.69 & $0=53$ & $0=50$ & $0=51$ & $0=32$ \\
& & $1=24$ & $1=21$ & $1=17$ & $1=18$ \\
& $2=4$ & $2=9$ & $2=13$ & $2=13$ \\
& & $3=9$ & $3=10$ & $3=9$ & $3=27$ \\
\hline Masculino (n=110) & 61,55 & $0=44$ & $0=16$ & $0=19$ & $0=9$ \\
& & $1=28$ & $1=41$ & $1=41$ & $1=14$ \\
& & $2=11$ & $2=22$ & $2=18$ & $2=34$ \\
& $3=27$ & $3=31$ & $3=32$ & $3=53$ \\
\hline
\end{tabular}

Nos dados descritos na tabela 3 podemos observar que em $48,5 \%, 35 \%$ e $33 \%$ dos pacientes apresentaram visualização insatisfatória do osso temporal contralateral, mesencéfalo e asa menor do esfenoide, respectivamente. 
Tabela 4: Frequência da categorização das variáveis ao modo B, osso temporal, mesencéfalo, asa do esfenóide, na população estudada.

\begin{tabular}{|cccc|} 
Pontuação & Temporal & Mesencéfalo & Esfenóide \\
& n (\%) & n $(\%)$ & n $(\%)$ \\
\hline $\mathbf{0}$ & $97(48,5)$ & $70(35)$ & $66(33)$ \\
\hline $\mathbf{1}$ & $52(26)$ & $58(29)$ & $62(31)$ \\
\hline $\mathbf{2}$ & $15(7,5)$ & $31(15,5)$ & $31(15,5)$ \\
\hline $\mathbf{3}$ & $36(18)$ & $41(20,5)$ & $41(20,5)$ \\
\hline
\end{tabular}

$\mathrm{Na}$ tabela abaixo, encontram-se as principais análises comparativas dos fatores capazes de predizer, isoladamente, a presença ou ausência da artéria cerebral média. Observa-se que idade e escore modo B apresentaram significância estatística $(p<0,001)$.

Tabela 5. Análise univariada para preditores de ACM visualizável.

\begin{tabular}{|lccl} 
Variáveis & ACM ausente & ACM presente & P-valor \\
& $\mathbf{n = 4 1}$ & $\mathbf{n = 1 5 9}$ & \\
Sexo masculino & $9(22 \%)$ & $101(62,5 \%)$ & $<0,001$ \\
Hipertensão & $36(87,8 \%)$ & $126(79,2 \%)$ & 0,268 \\
Diabetes & $12(29,3 \%)$ & $46(28,9 \%)$ & 0,99 \\
Pele negra & $10(24,4 \%)$ & $31(19,5 \%)$ & 0,517 \\
dislipidemia & $25(61 \%)$ & $92(57,9 \%)$ & 0,859 \\
idade & $70,54 \pm 12,7$ & $58,75 \pm 14,9$ & $<0,001$ \\
Escore modo B & $0,46 \pm 1,027$ & $4,16 \pm 3,003$ & $<0,001$ \\
\hline
\end{tabular}


$\mathrm{Na}$ análise multivariada a idade e o Escore modo $\mathrm{B}$ foram preditores independentes da presença de sinal visualizável da ACM, tabela 6 e 7. Na tabela 6 observa-se que a idade a pontuação relacionada a visualização da asa menor do esfenoide e do mesencéfalo são os únicos preditores independentes de visualização do fluxo em artéria cerebral médica pelo Doppler colorido (Tabela 6).

Tabela 6. Análise multivariada para preditores independentes de ACM visualizável no duplex transcraniano.

\begin{tabular}{|lccc|}
\hline Variável & OR & I C 95\% & P-valor \\
\hline Idade & 0,95 & $0,91-0,99$ & 0,007 \\
Asa menor do esfenóide & 13,76 & $2,99-63,42$ & 0,001 \\
Mesencéfalo & 6,6 & $1,59-27,87$ & 0,009 \\
\hline
\end{tabular}

Na tabela 7, em que o escore global do Modo B foi incluído no modelo de regressão logística multivariada ao invés dos componentes individuais que foram o escore, observa-se que a idade e a pontuação global no escore do Modo B relacionada são os únicos preditores independentes de visualização do fluxo em artéria cerebral médica pelo Doppler colorido (Tabela 7).

Tabela 7. Análise multivariada para preditores independentes de ACM visualizável no duplex transcraniano incluindo o Escore do Modo B.

\begin{tabular}{|llll|}
\hline Variável & OR & I C 95\% & P-Value \\
\hline Idade & 0,95 & $0,92-0,99$ & 0,011 \\
Escore modo B & 2,97 & $1,93-4,55$ & $<0,001$ \\
\hline
\end{tabular}




\section{3 - Avaliação da acurácia do Escore de modo B}

Observamos uma boa acurácia do escore do modo B como preditor de uma janela intracraniana satisfatória para visualização do sinal na ACM ispsilateral conforme evidenciado por uma área sob a curva $(\mathrm{AUC}=0.896 ; \mathrm{p}<0,001)$ na estatística $\mathrm{C}$ da curva ROC representada pela figura abaixo (figura 7).

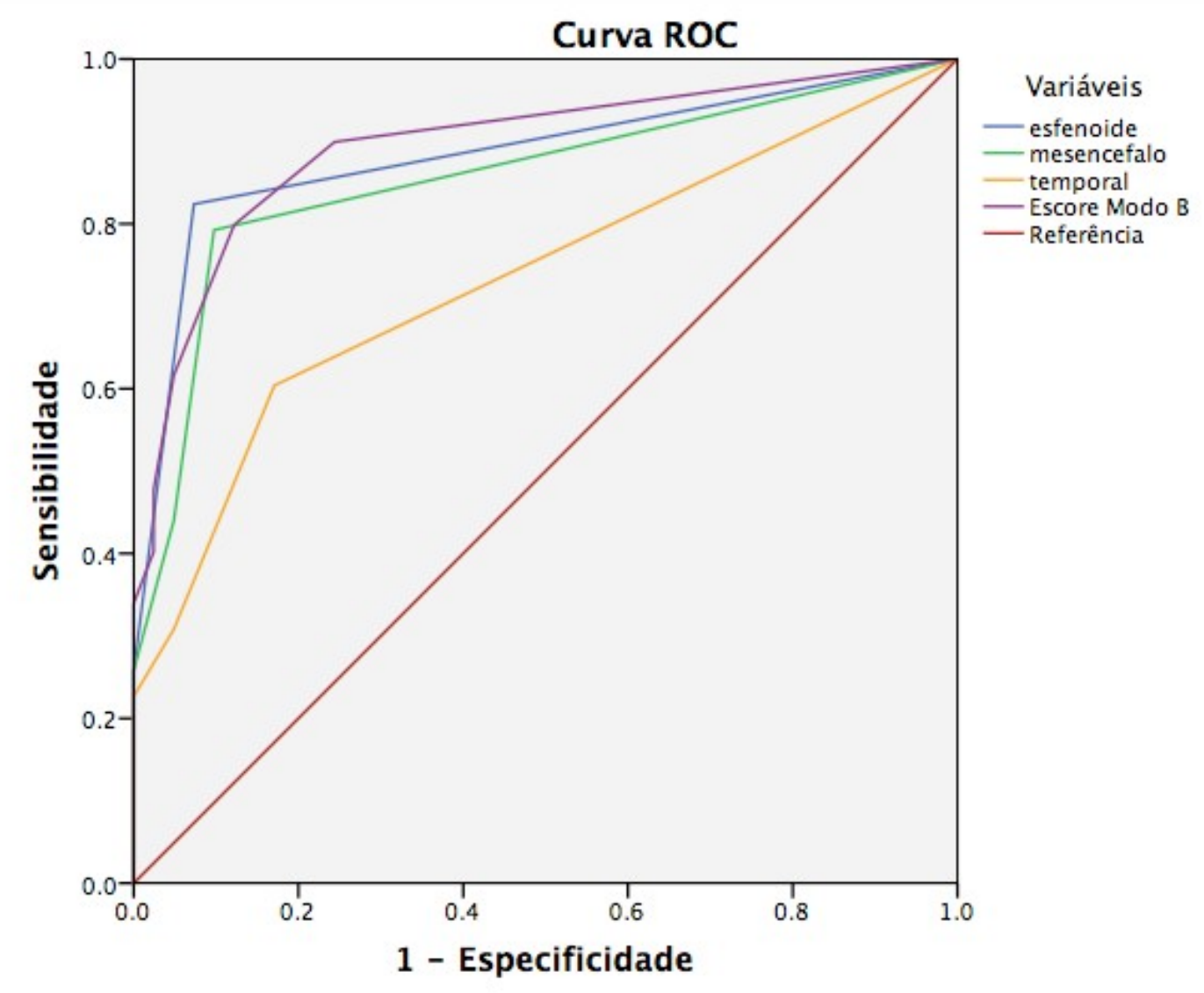

Diagonal segments are produced by ties.

Figura 7: Gráfico da curva ROC para a pontuação atribuída a visualização da asa menor do esfenoide, osso temporal contralateral, mesencéfalo e ao escore do modo B, como preditores da visualização do sinal do fluxo da artéria cerebral média no Doppler colorido. 
Tabela 8. Área sob a curva relacionada a curva ROC de preditores da visualização do fluxo na artéria cerebral média.

\begin{tabular}{|lcc|}
\hline Variáveis & Área sob a curva & p-valor \\
\hline Asa menor do esfenóide & $\mathbf{0 , 8 8 5}$ & $<\mathbf{0 , 0 0 1}$ \\
\hline Mesencéfalo & $\mathbf{0 , 8 5 6}$ & $<\mathbf{0 , 0 0 1}$ \\
\hline Osso temporal contralateral & $\mathbf{0 , 7 3 4}$ & $<\mathbf{0 , 0 0 1}$ \\
\hline Escore do Modo B & $\mathbf{0 , 8 9 6}$ & $<\mathbf{0 , 0 0 1}$ \\
\hline
\end{tabular}




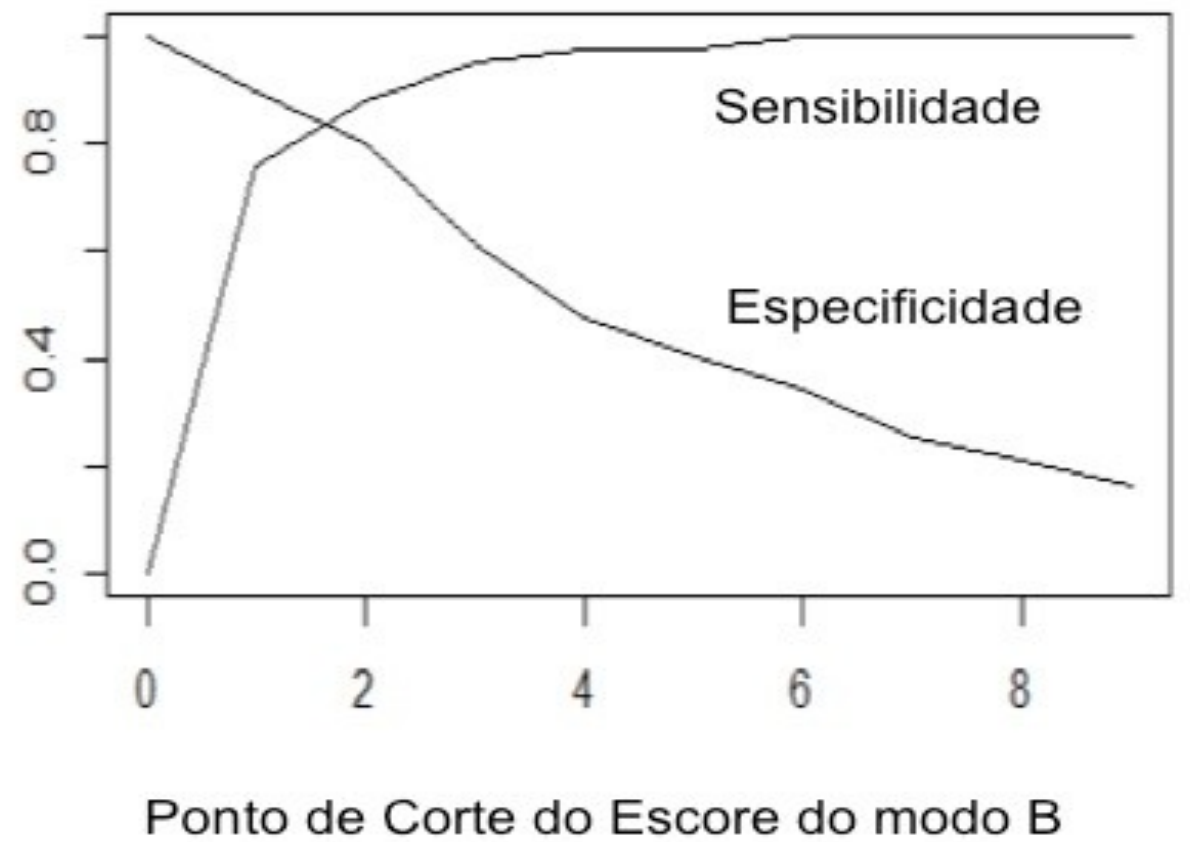

Figura 8: Análise de sensibilidade para determinação do ponto operador ótimo para o escore do modo B como preditor da visualização do sinal da artéria cerebral média no Doppler colorido.

A figura 8 acima elaborada para análise de sensibilidade do escore do Modo B para determinação do ponto operador ótimo do escore, sugere que o ponto de corte de 3 parece ser o ponto com maior acurácia do escore. Aplicando este ponto de corte teremos uma sensibilidade de $80 \%$ e uma especifidade de $87 \%$ para uma janela adequada a visualização do sinal da ACM ao UTC. 


\section{Discussão}

Este é o primeiro estudo que propõe a avaliação sistemática e semi-quantitativa da qualidade de visualização dos marcos anatômicos pelo modo B do UTC como forma de classificar a janela transtemporal em pacientes com AVC agudo. Nosso resultados sugerem que a análise sistemática e estruturada da qualidade de visualização das referencias anatômicas pelo modo B pode ser um bom métodos para estimativa da qualidade da avaliação ultrassonográfica subsequente do fluxo das artérias intracranianas pelo Doppler colorido, sobretudo da ACM ipsilateral.

Os dados da literatura mostram que a falência no estudo hemodinâmico através da janela transtemporal é significativamente mais comum em mulheres e aumenta com a idade. ${ }^{15}$ Em nossa casuística, as mulheres foram mais jovens que os homens mas apresentam menor taxa de sucesso na identificação da asa do esfenóide bem como pior sinal de visualização da primeira porção da ACM. À análise univariada, o gênero masculino apresentou forte associação com boa visualização do sinal da $\operatorname{ACM}(\mathrm{p}<0,001)$.

Esses resultados se alinham aos de Wijnhoud et al. (2008), em que o gênero foi uma variável importante para a qualificação da janela transtemporal. ${ }^{16} \mathrm{Nele}$, mulheres com 80 anos ou mais apresentaram janela transtemporal considerada ótima em $46,1 \%$ dos casos, comparada com a taxa de $72,7 \%$ nos homens de mesma faixa etária. ${ }^{16}$ A idade afeta mais severamente a detecção de sinal sonográfico ideal na população feminina. Essa diferença encontrada na população feminina, neste estudo, foi explicada pela maior espessura e não- homogeneidade do osso temporal, comum na osteoporose. ${ }^{17}$ 
Em paralelo, Jo'zsefKollár et al (2004), organizaram um estudo multicêntrico com 33 pacientes neurológicos moribundos. Investigou-se a relação entre a qualidade acústica da janela transtemporal desses pacientes vivos e a espessura, densidade e homogeneidade do osso temporal estimado pela tomografia computadorizada, pós-morte. Surpreendentemente, demonstraram que a não-homogeneidade do osso não está associada à espessura do osso, nem à qualidade acústica da janela transtemporal. ${ }^{18}$

Segundo Silvidi et al. (1988), possivelmente a dificuldade de insonação através da janela transtemporal está relacionada à hiperostose do osso temporal, influenciada além do gênero e idade, pela etnia. ${ }^{19}$ No entanto, não é incomum, na prática clínica não se obter sinal sonográfico arterial à despeito de uma janela acústica transtemporal penetrável, em decorrência de severa hipoplasia ou aplasia das artérias cerebrais. ${ }^{20}$ A aplasia da artéria cerebral média ou da basilar é extremamente rara, mas aplasia ou hipoplasia da artéria cerebral anterior ou vertebral, pode ser encontrada de $1 \%$ a $5 \%$ da população. ${ }^{18-20}$

Entretanto, Baumgartner et al. (1994) mostraram que a taxa de detecção das artérias intracranianas diminui com o aumento da idade, mas não encontrou diferença significativa entre homens e mulheres, provavelmente pelo número reduzido de pacientes $(n=33) .{ }^{21} \mathrm{Em}$ nosso estudo, a idade foi um fator que mostrou associação significativa para predizer a visualização da $\mathrm{ACM}(\mathrm{p}<0,001)$.

Com relação as características étnicas da população estudada, a maioria foi considerada branca $(\mathrm{n}=159 ; 79,5 \%)$. A análise individual da cor da pele negra não obteve associação para predizer a visualização da $\mathrm{ACM}(\mathrm{p}=0,517)$. Estes achados podem ser explicados pela fragilidade da auto-classificação da cor da pele utilizada no presente estudo, que foi derivada da própria classificação utilizada para registro em nossa Instituição.

Em populações caucasianas européias, estudos demonstraram janela transtemporal 
inapropriada em 5 a $8 \%$ dos indivíduos ${ }^{22}$. Dados similares foram encontrados em estudo chileno, com falência acústica transtemporal em 5\% dos pacientes, vale ressaltar a predominância de descendentes europeus nesta população. ${ }^{23}$ Esta taxa de falência da janela transtemporal sobe para $23 \%$ nos homens e $50 \%$ nas mulheres de grupos étnicos miscigenados, com contribuições africanas e asiáticas. ${ }^{24,25}$

Encontramos que dentre as estruturas anatômicas intracranianas usualmente visualizadas através da janela transtemporal no Modo B (asa menor do esfenoide, mesencéfalo e osso temporal contra-lateral) a asa menor do esfenoide e o mesencéfalo tem o maior valor para estimativa da qualidade da janela transtemporal ipsilateral. De fato, a visualização do osso temporal contra-lateral não foi um parâmetro independentemente associado a visualização do sinal da ACM no UTC.

Este estudo apresentou limitações importantes, quais sejam, dificuldade em estabelecer a qualidade da janela transtemporal de acordo com a etnia/raça (miscigenação brasileira), presente em outros estudos. O exame foi realizado somente em indivíduos admitidos com suspeita de AVC, o que pode refletir num viés de seleção. Outras condições como insuficiência renal ou pancreática, que já são previamente associadas à janela transtemporal subótima não foram analisadas se o efeito de transdutores com frequência menor que $2 \mathrm{MHz}$ aumenta a detecção de sinal das artérias proximais numa janela acústica inadequada. Adicionalmente, nos limitamos a estudas a janela transtemporal, que é a mais utilizada. Nossos resultados não podem ser extrapolados para outras janelas transcranianas. 


\section{Conclusões}

Os resultados do presente estudo possibilitam concluir que:

1. A frequência de visualização da osso temporal contralateral, asa menor do esfenoide ipsilateral, e mesencéfalo como pontos de referencia anatômica intracraniana pelo modo B do UTC é:

- Osso temporal contralateral: sinal insatisfatório (48\%), pobre (26\%), satisfatório $(7,5 \%)$, ótimo $(18 \%)$.

- Mesencéfalo: sinal insatisfatório (35\%), pobre (29\%), satisfatório $(15,5 \%)$, ótimo $(20,5 \%)$

- Asa menor do esfenóide ipsilateral: sinal insatisfatório (33\%), pobre (31\%), satisfatório $(15,5 \%)$, ótimo $(20,5 \%)$.

2. Criamos um escore de modo B que baseado na qualidade de visualização do osso temporal contralateral, asa menor do esfenoide ipsilateral, e mesencéfalo como os marcos anatômicos visualizados no modo $\mathrm{B}$ (de 0 a 9) que permite prever a capacidade de insonação adequada da ACM com elevada acurácia (área sob a curva $\mathrm{ROC}=0,89 ; \mathrm{p}<0.001)$.

3. A idade dos paciente e o sua pontuação no Escore modo B são preditores independentes da presença de sinal visualizável da ACM ao UTC na fase aguda do AVC. 


\section{Referências}

1. Allan PL, Dubbins AP, Pozniak MA, McDicken WN. Clinical Doppler ultrasound. Philadelphia: Churchill Livingstone, 2000, p.1-293.

2. Baumgartner RW, Mattle HP, Aaslid R. Transcranial color-coded duplex sonography, magnetic resonance angiography, and computed tomography angiography: methods, applications, advantages, and limitations. J Clin Ultrasound [Internet]. 1995 Feb [cited 2015 Nov 16];23(2):89-111.

3. R. Aaslid, T. M. Markwalder, and H. Nornes, "Noninvasive transcranial Doppler ultrasound recording of flow velocity in basal cerebral arteries," Journal of Neurosurgery, vol. 57, no. 6, pp.769-774,1982.

4. Naqvi J, Yap KH, Ahmad G, Ghosh J. Transcranial Doppler ultrasound: a review of the physical principles and major applications in critical care. Int J Vasc Med Volume 2013.

5. G.Tsivgoulis,A.V.Alexandrov,andM.A.Sloan,“Advances in transcranial Doppler ultrasonography,"Current Neurology and Neuroscience Reports,vol.9,no.1,pp.46$54,2009$.

6. H. A. Nicoletto and M. H. Burkman, "Transcranial Doppler series part II: performing a transcranial Doppler," American Journal of Electroneurodiagnostic Technology, vol. 49, no. 1, pp. 14-27,2009. 
7. Fujioka, Douville CM. Anatomy and freehand examination techniques. In: Aaslid R, Newell DW. Transcranial Doppler, New York: Raven Press, 1992. p.9-31.

8. Berland LL, Bryan CR, Sekar BC, Moss CN. Sonographic examination of the adult brain. J Clin Ultrasound [Internet]. 1988 Jun [cited 2015 Nov 16];16(5):337-45. Available from: http://doi.wiley.com/10.1002/jcu.1870160508.

9. Bartels E. Transcranial color-coded duplex ultrasonography in routine cerebrovascular diagnostics. Perspect Med [Internet]. Elsevier GmbH.; 2012;1- 12(112):325-30. Available from: http://dx.doi.org/10.1016/j.permed.2012.06.001.

10. Schöning M, Grunert D, Stier B. [Transcranial duplex sonography through intact bone: a new diagnostic procedure]. Ultraschall Med [Internet]. 1989 Apr 1 [cited 2015 Nov 16];10(2):66-71. Available from: http://www.researchgate.net/publication/245886042_Transkranielle_Duplexsonogr aph ie_durch_den_intakten_Knochen_Ein_neues_diagnostische_Verfahren

11. Halsey, J. H. 1990. Effect of emitted power on waveform intensity in transcranial Doppler. Stroke 21:1573-1578.

12. Seidel G, Kaps M, Gerriets T. Potential and limitations of transcranial color-coded sonography in stroke patients. Stroke. 1995;26:2061-2066

13. Krejza J, Swiat M, Pawlak MA, Oszkinis G, Weigele J, Hurst RW, Kasner S. Suitability of temporal bone acoustic window: conventional tcd versus transcranial color-coded duplex sonography. J Neuroimaging. 2007;17:311-314

14. Lange MC, Zétola VH, Miranda-Alves $\mathrm{M}$, Moro $\mathrm{CH}$, Silvado $\mathrm{CE}$, Rodrigues DL,Gregorio EG, Silva GS, Oliveira-Filho J, Perdatella MT, Pontes-Neto OM, Fábio SR,Avelar WM, Freitas GR; Task Force Group of the Neurosonology Department,Brazilian Academy of Neurology. Brazilian guidelines for the application of transcranial ultrasound as a diagnostic test for the confirmation of 
brain death. Arq Neuropsiquiatr. 2012 May;70(5):373-80. PubMed PMID: 22618790 .

15. Kwon J-H, Kim JS, Kang D-W, Bae K-S, Kwon SU. The Thickness and Texture of Temporal Bone in Brain CT Predict Acoustic Window Failure of Transcranial Doppler.J Neuroimaging 2006;16:347-352.

16. Wijnhoud, A. D., M. Franckena, A. van der Lugt, P. J. Koudstaal, and E. D. Dippel. 2008. Inadequate acoustical temporal bone window in patients with a transient ischemic attack or minor stroke: role of skull thickness and bone density. Ultrasound Med. Biol. 34:923-929.

17. Kwon, J. H., J. S. Kim, D. W. Kang, K. S. Bae, and S. U. Kwon. 2006. The thickness and texture of temporal bone in brain CT predict acoustic window failure of transcranial Doppler. J. Neuroimaging 16:347-352

18. Jo'zsefKolla'ra Gernot Schulte-Altedorneburgb,d Judit Sikulaa BélaFülesdic E. BerndRingelsteind Vineet Mehtaa La'szlo' Csibab DirkW.Drosted,e Cerebrovasc Dis 2004;17:61-65 DOI: 10.1159/000073899

19. Bruno A, Biller J, Silvidi JA. A reason for failure to obtain transcranial Doppler flow signals: hyperostosis of the skull. Stroke. 1988;19:274. Letter. 8. Halsey JH. Effect of emitted power on waveform intensity in transcranial Doppler. Stroke. 1990;21:1573-1578.

20. Riggs HE, Rupp C. Variation in form of circle of Willis: the relation of the variations to collateral circulation: anatomic analysis. Arch Neurol. 1963;8:8-1

21. Baumgartner RW, Mattle HP, Kothbauer K, Schroth G. Transcranial color-coded duplex sonography in cerebral aneurysms. Stroke. 1994;25: 2429-2434. 
22. Ogata T, Kimura K, Nakajima M, Naritomi H, Minematsu K. Diagnosis of middle cerebral artery occlusive lesions with contrast-enhanced transcranial color-coded real-time sonography in acute stroke. Neuroradiology. 2005;47:256-262.

23. . M. A. Topcuoglu, "Transcranial Doppler ultrasound in neurovascular diseases: diagnostic and therapeutic aspects," Journal of Neurochemistry, vol.123, supplement 2, pp.39-51, 2012.

24. Brunser AM, Silva C, Cárcamo D, Muñoz P, Hoppe A, Olavarría VV, Díaz V, Abarca J. Transcranial Doppler in a Hispanic-Mestizo population with neurological diseases: a study of sonographic window and its determinants. Brain Behav. 2012 May;2(3):231-6.

25. E. C. Jauch, J. L. Saver, H. P. Adams et al., "Guidelines for the early management of patients with acute ischemic stroke: a guideline for health care professionals from the American Heart Association/American Stroke Association," Stroke, vol. 44, pp. 870-947, 2013. 


\section{Anexos}

ANEXO A -Aprovação no Comitê de Ética em Pesquisa

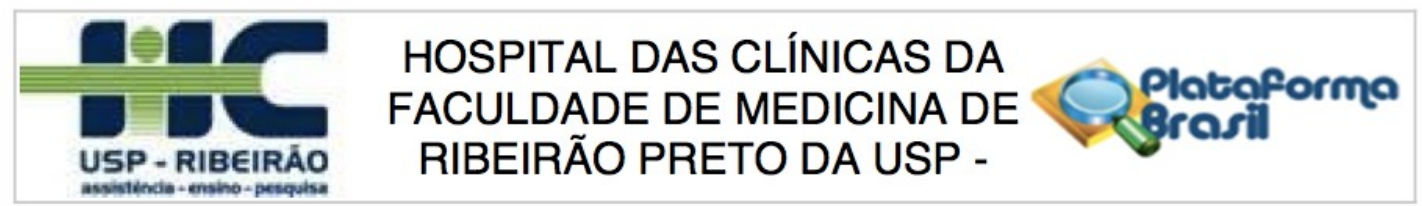

\section{PARECER CONSUBSTANCIADO DO CEP}

\section{DADOS DO PROJETO DE PESQUISA}

Título da Pesquisa: Avaliação da frequência e gravidade da Doença Aterosclerótica Intracraniana nos pacientes com AVCi ou AIT através da ultrassonografia transcraniana colorida e angiotomografia de crânio

Pesquisador: Octavio Marques Pontes Neto

Área Temática:

Versão: 2

CAAE: 32115414.0 .0000 .5440

Instituição Proponente:Hospital das Clínicas da Faculdade de Medicina de Ribeirão Preto da USP -

Patrocinador Principal: Financiamento Próprio

DADOS DO PARECER

Número do Parecer: 737.008

Data da Relatoria: 25/07/2014 


\title{
ANEXO B - Termo de consentimento livre e esclarecido
}

\author{
TERM O DE CONSENTIM ENTO LIVRE E ESCLARECIDO - TCLE \\ hospital das Clínicas da FaCuldade de Medicina de Ribeirão Preto \\ UNiVERsidade de SÃo PAUlo (FMRP- USP)
}

\section{INFORMAÇÃO AOS SUJEITOS DE PESQUISA E TERMO DE CONSENTIMENTO LIVRE E ESCLARECIDO}

TÍTULO DO PROJETO: Avaliação da frequência e gravidade da Doença Aterosclerótica Intracraniana nos pacientes com AVCi e AIT através da ultrassonografia transcraniana colorida e angiotomografia de crânio

INVESTIGADOR PRINCIPAL: Octávio Marques Pontes Neto (CRM: 98293)

PESQUISADORES COLABORADORES: Renata da Silva Almeida Santos, Letícia Januzi de Almeida Rocha, Pedro Telles Cougo Pinto, Millene Rodrigues Camilo, Frederico Fernandes Alessio Alves, Clara Monteiro Barreira, Francisco Antunes Dias.

INSTITUIÇÃO: Faculdade de Medicina de Ribeirão Preto

O(A) Senhor(a), está sendo convidado(a) a participar de um estudo que tem por objetivo avaliar a frequência e gravidade da aterosclerose intracraniana (entupimento nos vasos do cérebro) nos pacientes em pacientes com Acidente Vascular Cerebral (AVC) Isquêmico.

O Acidente Vascular Cerebral isquêmico é uma doença grave que é causada pelo entupimento de um vaso sanguíneo cerebral. De uma maneira geral, o AVC é a principal causa de morte no Brasil e também a principal causa de invalidez não traumática (não causada por acidente) e pode estar associado ou ser causado por diversas condições clínicas, incluindo a aterosclerose intracraniana, que é um estreitamento no vaso sanguíneo. O tratamento de cada tipo de AVC deve ser individualizado, e isso só é possível quando sua causa é conhecida.

Através deste estudo nós pretendemos avaliar a frequência de estreitamento dos vasos intracranianos nos pacientes de AVCi internados na Unidade de Emergência do Hospital das Clínicas de Ribeirão Preto. Para tal, o senhor(a) será submetido(a) a um exame de ultrassonagrafia transcraniana que será realizado por um Neurologista treinado da equipe. O exame de Ultrassonografia transcraniana é seguro e não causa dor, e faz parte da investigação das causas de AVC.

Você será avaliado por um dos pesquisadores e serão colhidas diversas informações clínicas em um questionário de pesquisa específico. Além disso, solicitamos 
sua autorização para ter acesso a dados do seu prontuário, assim como resultado de exames realizados durante seu internamento ou acompanhamento ambulatorial.

Neste estudo você não terá modificação no seu tratamento, ou seja, não serão testadas medicações ou novos tratamentos. Não existe custo algum para você ou sua família, assim como não há remuneração ou distribuição de medicações em troca de sua participação.

As informações obtidas são confidenciais e só serão utilizadas para fins de pesquisa. A sua participação é voluntária e o seu atendimento não será prejudicado caso prefira não participar, sendo assim o seu consentimento pode ser retirado a qualquer momento do estudo, ou seja, você pode desistir sem prejuízo ao seu tratamento.

Apesar de não the trazer benefícios diretos, sua participação em nossa pesquisa pode nos ajudar a identificar o estreitamento dos vasos cerebrais através de um exame mais simples e barato, que é o ultrassonografia dos vasos intracranianos, podendo ajudar outras pessoas no futuro.

Qualquer intercorrência relacionada aos procedimentos deste estudo será de responsabilidade do investigador responsável e você terá toda assistência médica necessária nesta instituição até a solução deste problema. Qualquer informação adicional poderá ser fornecida em qualquer fase da pesquisa pelo pesquisador responsável através do telefone (16) 99796-7346 ou na Unidade de Pesquisa Clínica da Unidade de Emergência do Hospital das Clínicas da Faculdade de Medicina de Ribeirão Preto, no número (16) 3602-1296. Apresente sua dúvida e deixe suas informações de contato. A Unidade de Pesquisa Clínica encaminhará suas questões para a equipe do estudo, que entrará em contato para resolvê-las o mais breve possível.

Após ter sido esclarecido(a) sobre o estudo e o que representa a minha participação concordo em participar.

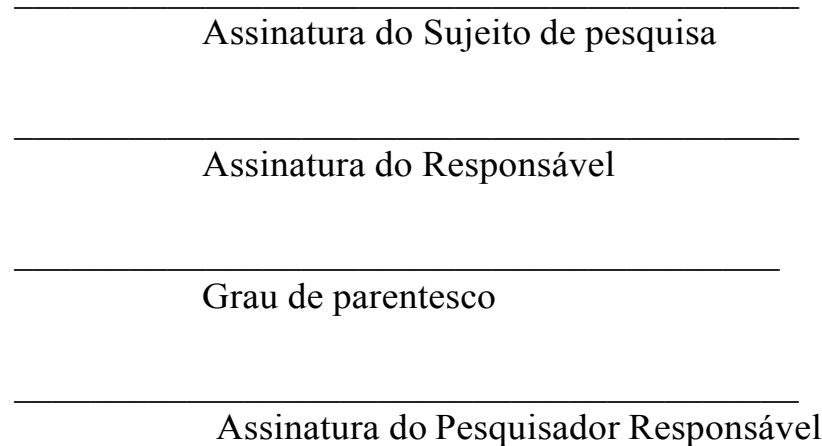

Assinatura do Pesquisador Responsável

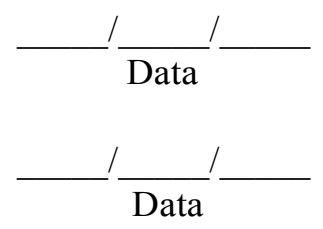

RG

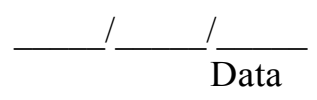

